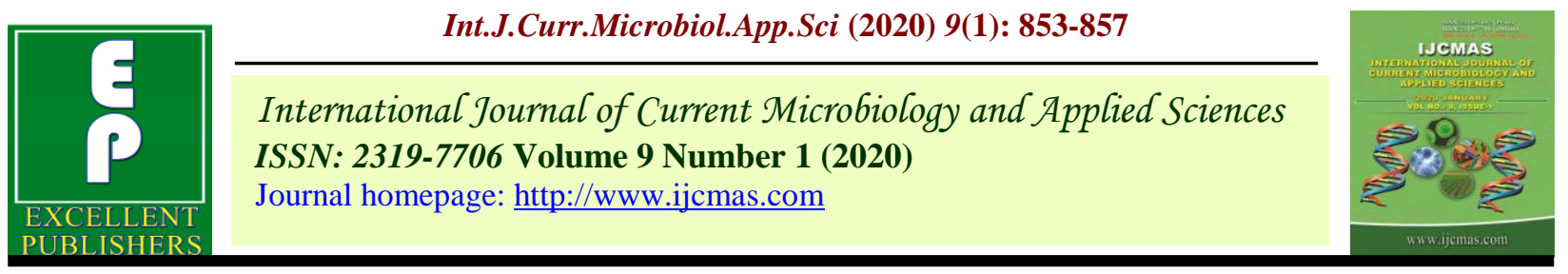

Original Research Article

https://doi.org/10.20546/ijcmas.2020.901.094

\title{
Comparison of in vitro Activities of Ceftazidime-Tazobactam, Piperacillin- Tazobactam, and Cefoperazone-Sulbactam and the Implication on Therapy in a Tertiary Care Teaching Hospital
}

\author{
E. Subbalakshmi ${ }^{1 *}$, H. Kalavathy Victor ${ }^{1}$ and K. Loganathan ${ }^{2}$ \\ ${ }^{1}$ Department of Microbiology, ${ }^{2}$ Department of Surgery, ACS Medical College and Hospital, \\ Chennai, Tamil Nadu, India \\ *Corresponding author
}

\begin{tabular}{|c|}
\hline Keywords \\
\hline $\begin{array}{l}\text { Ceftazidime- } \\
\text { Tazobactam, } \\
\text { Piperacillin- } \\
\text { Tazobactam and } \\
\text { Cefoperazone- } \\
\text { Sulbactam }\end{array}$ \\
\hline Article Info \\
\hline $\begin{array}{l}\text { Accepted: } \\
15 \text { December } 2019 \\
\text { Available Online: } \\
20 \text { January } 2020\end{array}$ \\
\hline
\end{tabular}

A B S T R A C T

Resistance in pathogenic bacteria against antibiotics is a challenge for our clinicians for the management of various infections. The emergence of antibiotic resistance has caused great concern among health professionals and the prevalence of multidrug-resistant organisms has been increasing. Over-ambitious use of broad spectrum antibiotics is playing an important role in the increased incidence of infections due to MDR bacteria. Multiple drug resistance (MDR) mediated through $\mathrm{R}$ plasmids among Gram-negative bacteria has become a major nosocomial problem worldwide. Broad-spectrum $\beta$-lactams, such as imipenem, cefdinir, cefepime and cefpirome, and $\beta$-lactamase inhibitor combinations, such as piperacillin/tazobactam, cefoperazone/sulbactam and ticarcillin/clavulanate have been introduced in the market to overcome this resistance. Aim: To compare the antibiotic sensitivity of piperacillin/tazobactam, ceftazidime/tazobactam and cefoperazone/ sulbactam for gram negative isolates in our institute from various samples received in the hospital. This study was aimed at identifying the possible etiologic agents and determining their antibiotic sensitivity pattern, thereby allowing formulation of a reasonable initial empirical therapy regimen. OBJECTIVE: To choose the optimal antibiotic as the treatment for our patients who are developing infections. Overall, the sensitivity to ceftazidime/tazobactum was much higher compared to cefoperazone/sulbactum, piperacillin/tazobactum. The sensitivity was observed in medical wards for CAZ/TAZ (81.3\%), CFS/SUL (49\%), PIP/TAZ (47.4\%), whereas in surgical wards the sensitivity observed was CAZ/TAZ (66\%), CFS/SUL (32\%), PIP/TAZ (27\%) respectively. The sensitivity to Enterobacteriaceae group was CAZ/TAZ (69.3\%), CFS/SUL (67\%), PIP/TAZ (59\%). The sensitivity to nonfermenters were CAZ/TAZ (31\%),CFS/SUL (33\%), PIP/TAZ (41\%). Among nonfermenters, the sensitivity to PIP /TAZ (41\%) was much better than CAZ/TAZ. Beta lactam inhibition combinations are used more in surgical patients than medical patients and the sensitivity to these combination drugs decreases with increased usage which clearly shows multidrug resistant pattern of organism. This can be prevented by following antibiotic policy and hand hygiene practice to avoid multidrug resistant organisms.

\section{Introduction}

Anti microbial resistance constitutes one of the major threats which are related to the pathogenic microorganisms. In any community or nosocomial setting, widespread antibiotic usage influences the prevalence and distribution of antibiotic resistance in 
common pathogens. ${ }^{[1]}$ Gram-negative pathogens such as Enterobacteriaceae (especially those which produce the extendedspectrum $\quad \beta$-lactamases), Pseudomonas aeruginosa, and Acinetobacter baumannii have acquired an important role in the nosocomial infections, which is of particular concern, because of the associated broad spectrum of the antibiotic resistance. The emergence of antibiotic resistance has caused great concern among health professionals due to increased prevalence of multidrug-resistant organisms. Multidrug-resistant organisms (MDROs) are labelled as such because of their in vitro resistance to more than one antimicrobial agent. ${ }^{[2]}$ Treatment of infectious diseases have become more challenging with each passing year. It is estimated that $80 \%$ of all hospital deaths are directly or indirectly related to HAIs. HAI are most commonly associated with lower respiratory tract infections, urinary tract infections, pneumonia, wound infections, bloodstream infections, surgical site infection (SSI) and sepsis which are primarily caused by a range of gram-negative organisms particularly E. coli, Acinetobacter spp, Klebsiella spp., Pseudomonas spp., Enterobacter spp. In any community or nosocomial setting, widespread antibiotic usage influences the prevalence and distribution of antibiotic resistance in common pathogen. Multiple drug resistance (MDR) mediated through $\mathrm{R}$ plasmids among Gram-negative bacteria has become a major nosocomial problem worldwide. ${ }^{[3]}$ Due to multiple drug resistance to $\beta$-lactams, aminoglycosides and quinolones, antimicrobial treatment of nosocomial infections caused by these bacteria is compromised ${ }^{[4]}$. Among the $\beta$-lactams, third generation cephalosporins, such as ceftazidime, cefotaxime, and ceftriaxone are routinely used in our clinical setting, and resistance to these drugs, due to $\beta$-lactamase production is frequently observed. ${ }^{[5]}$ Broadspectrum $\beta$-lactams, such as imipenem, cefdinir, cefepime and cefpirome, and $\beta$ lactamase inhibitor combinations, such as piperacillin/tazobactam, cefoperazone/ sulbactam and ticarcillin/clavulanate, have been introduced in the market to overcome this resistance. With the development of new antimicrobial agents, bacteria too are developing resistance against these newer agents. We measured the degree of in vitro activity of these new $\beta$-lactam drugs against clinical isolates belonging to the family Enterobacteriaceae, Pseudomonas spp. and Acinetobacter species, which were resistant to routinely used third generation cephalosporins, such as ceftazidime, cefotaxime and ceftriaxone. The present study was done to compare the in vitro activity of combination of beta lactamase inhibitor combination on the inpatients samples in a tertiary care teaching hospital.

\section{Materials and Methods}

This study was done in our institute from Jan 2019 to June 2019. The study was a prospective study and the samples were collected from all the inpatients of the hospital. All the age group of the patients were included in the study. We did not include out patients in our study. Ethical clearance was obtained from the Institute before starting the study. Out of 1154 isolates 100 samples which were multidrug resistant were taken for the further study. Various clinical samples included sputum, pus, urine, blood and other body fluids (CSF, pleural fluid, BAL etc.) that were received at the Microbiology department. The samples were processed, and the strains were identified and characterized by the following tests. Gram stain, oxidase test, catalase test, motility by both hanging drop and semi-solid agar methods, Hugh \& Leifson $\mathrm{O} / \mathrm{F}$ test, citrate utilization, urease production, nitrate reduction, indole production, phenylpyruvic acid production, pigment production, lysine \& 
ornithine decarboxylation, arginine dihydrolase test, and oxidation of $1 \%$ glucose, lactose, sucrose and mannitol. The antibiotic sensitivity was determined by means of the Kirby Baeur disc diffusion method, according to CLSI ( Clinical Laboratory Standard Institute) guide lines 2019. ${ }^{[6]}$ The strains that were resistant to ceftazidime, cefotaxime, ceftriaxone were tested for our further study. Extended Spectrum BetaLactamase (ESBL) production was confirmed by CLSI recommendations using cephalosporinclavulanate combination discs. A difference of $>5 \mathrm{~mm}$ between the zone diameter of either of the cephalosporin discs and their respective cephalosporin-clavulanate discs was taken to be the phenotypic confirmation of ESBL production. We used betalactam inhibitor combination of tazobactam, sulbactam for our study. The combinations that were added in our study were ceftazidime, ceftazidime/tazobactam, piperacillin, piperacillin/tazobactam, cefoperazone, cefoperazone /sulbactam. All the abovementioned antimicrobial discs were obtained from Hi-media. ${ }^{[7]}$

\section{Statistical Methods}

Descriptive and inferential statistical analysis has been carried out in the present study. Results on continuous measurements are presented on Mean \pm SD (Min-Max) and results on categorical measurements are presented in Number (\%). Significance is assessed at $5 \%$ level of significance. The following assumptions on data is made, Assumptions: 1.Dependentvariables should be normally distributed, 2.Samples drawn from the population should be random, Cases of the samples should be independent

Chi-square/ Fisher Exact test has been used to find the significance of study parameters on categorical scale between two or more groups, Non-parametric setting for Qualitative data analysis. Fisher Exact test used when cell samples are very small.

\section{Results and Discussion}

Out of 1154 samples that were processed, the samples that were resistant to ceftazidime, cefotaxime and ceftriaxone were taken for study. Totally 100 multidrug resistant samples were taken for study. The samples that we received included 33 male patients and 67 female patients [Table -1]. The samples included urine 65 , sputum 12 , pus 20 and tissue 3 [Table 2]. We received 59 samples from various medical wards and 41 samples from different surgical wards. The organisms isolated were Acinetobacter spp. 10, Citrobacter spp 3, E. coli 49, Klebsiella spp 21. Pseudomonas spp.15, Proteus mirabilis 2 from various samples.

Table.1 Sample type distribution of patients studied

\begin{tabular}{|l|c|c|}
\hline \multicolumn{1}{|c|}{ Sample } & No. of patients & \% \\
\hline Pus & 20 & 20.0 \\
\hline Sputum & 12 & 12.0 \\
\hline Tissue & 3 & 3.0 \\
\hline Urine & 65 & 65.0 \\
\hline Total & 100 & 100.0 \\
\hline
\end{tabular}

After isolating the multidrug resistant isolates, we compared the antibiotics susceptibility pattern with regards to CAZ/CAZ/TAZ, CFS, CFS/SUL, PIP, PIP/TAZ. Overall the sensitivity of the organisms to the drugs CAZ/CEF/TAZ was high in both medical and surgical wards. Sensitivity being high for CEF/TAZ- 48 (81/.3\%), CFS/SUL- 29(49\%) and for PIP/TAZ -28 (47.4\%) from various medical wards where as in case of surgical wards the sensitivity for CAZ/TAZ -27 (66\%), CFS/SUL - 13 (32\%), PIP/TAZ $11(27 \%)$ respectively. The resistant to all the three drug combinations were high in surgical 
wards compared to medical ward samples because combination drugs are used mostly in surgical wards following surgeries. When we compared the sensitivity to pattern to organisms we found in case of Enterobacteriaceae, the sensitivity pattern to CAZ/TAZ -52 (69.3\%), CFS/SUL- 28 (67\%), PIP/TAZ -23 (59\%) whereas in case of nonfermenters, the sensitivity pattern to CAZ/TAZ- 23 (31\%), CFS/SUL- 14 (33\%), PIP/TAZ - 16 (41\%) respectively.

Table.2 Organism distribution of patients studied

\begin{tabular}{|l|c|c|}
\hline \multicolumn{1}{|c|}{ Organism } & No. of patients & $\%$ \\
\hline Acinetobacter $\mathrm{sp}$ & 10 & 10.0 \\
\hline Citrobacter $\mathrm{sp}$ & 3 & 3.0 \\
\hline E.coli & 49 & 49.0 \\
\hline Klebsiella $\mathrm{sp}$ & 21 & 21.0 \\
\hline Proteus mirabilis & 2 & 2.0 \\
\hline Pseudomonas $\mathrm{sp}$ & 15 & 15.0 \\
\hline Total & 100 & 100.0 \\
\hline
\end{tabular}

The present study has demonstrated CAZ/TAZ as the most effective beta lactam inhibitor combination antibiotic with respect to combination antibiotic. Owing to the changing resistance patterns and increased prevalence of $\beta$ lactamase producing strains, relevant pathogens may not be susceptible and therapy with a single antibiotic may promote antimicrobial resistance. Anuradha et al., ${ }^{[8]}$ have reported PIP/TAZ as the most active combination of the agent against Enterobacter, Pseudomonas spp but they found Ticarcillin/Clavulinic acid to be highly effective against Acinetobacter spp. And Sternotrophomonas maltophilia. In our study we found PIP/TAZ was effective against non fermenters than CAZ/TAZ. A study from Chandigarh says both CFS/SUL and PIP/TAZ as effective as combination against gram negative isolates. ${ }^{[9]} \mathrm{IN}$ as tudy on gram negative bacteria which were isolated form medical oncology patients, the activity of CFS/SUL were compared to that of PIP/TAZ. They found that CFS/SUL was found to efficient than PIP/TAZ. ${ }^{[10]}$ Studies in India have shown PIP/TAZ to be better than CFS/SUL especially against Pseudomonas sp, Klebsiella sp, and E. coli. ${ }^{[11,12]}$ Tazobactam is the most promising inhibitor which unlike sulbactam and clavulanic acid has its own antibacterial activity. Sulbactam containing combinations have not demonstrated strong selective pressures for ESBL producing Enterobacteriaceae. In contrast to clavulanate, sulbactam does not induce class I (Amp C) chromosomal beta-lactamases in Enterobacteriaceae. ${ }^{[13]}$ In Gupta et al., study Only $5.3 \%$ of the strains were resistant against cefoperazone/sulbactam. Various studies have shown that sulbactam increases the activity of cefoperazone against Enterobacteriaceae and nonfermenters. ${ }^{[14]}$ [Table 6]. We found CAZ/TAZ had better sensitivity than CFS/SUL. In our study we found CAZ/TAZ combination was sensitive to most of the organisms in the wards. There was not much of a difference with respect to CFS/SUL and PIP/TAZ/. The variation in the susceptibility rates of $\beta$ lactam/ $\beta$ lactamase inhibitor combination in different studies could be due to the difference in the hospital organism and also PIP/TAZ [Table 7] is the most commonly used drug in surgical wards. This could have resulted in a selection pressure for the development of resistance to the drug. The emergence of resistance in microbes can be prevented by implication of strict guidelines for antibiotic prescribing and appropriate infection control measures.

It is concluded that the present study was important from a practical point of view. The clinical and therapeutic implication of antibiotic resistance implies that strict guidelines for antibiotic prescribing and appropriate infection measures are required to prevent MDS strains. 


\section{Acknowledgements}

I thank my HOD, Dept of Microbiology for her support in the study and Dr.K.Loganathan, HOD, Dept of Surgery for the samples and support.

\section{References}

1.Perez-Liarena FJ, Bou G. Beta-Lactamase Inhibitors: The Story so Far. Curr Med Chem. 2009;16(28):3740-65.

2.Jones RN, Kirby JT, Beach ML, Biedenbach DJ, Pfaller MA. Geographic variations in activity of broad-spectrum betalactams against Pseudomonas aeruginosa: summary of the worldwide SENTRY Antimicrobial Surveillance Program (1997-2000). Diagn Microbiol Infect Dis. 2002; 43:239-43.

3.Ram S, Gupta R, Gaheer M, Uppal S. Prevalence of multiple drug resistant organisms in an intensive care burn unit. Indian J Med Res 2000: 118-20.

4.Gales A.C., Jones R.N., Turnidge J., et al., Characterization of Pseudomonas aeruginosa isolates: occurrence rates, antimicrobial susceptibility patterns and molecular typing in the global SENTRY antimicrobial surveillance program 1997-1999. Clin Infect Dis 2001;32(suppl 2): s146-55.

5.Mahapatra A, Samal B, Pattnaik D, et al., Antimicrobial susceptibility pattern of clinical isolates of non- fermentative bacteria. Indian $\mathbf{J}$ Pathol Microbiol 2003;46(3):526-7.

6.Clinical and Laboratory Standards Institute (CLSI). Performance standards for Antimicrobial Susceptibility Testing; Twenty eighth Informational supplement. M100-S23. Wayne, PA: CLSI, 2018.

7.Joshi etal, Piperacillin/tazobactam plus tobramycin versus ceftazidime plus tobramycin for the treatment of patients with nosocomial lower respiratory tract infection Journal of Antimicrobial Chemotherapy (1999) 43, 389-397.

8. Anuradha V, Sailaja V V, Umabala P, Sateesh T, Lakshmi V. Sensitivity pattern of Gram negative Bacilli to three $\beta$-lactam/ $\beta$-lactamse inhibitor combinations using the automated API system. Ind J Med Microbiol. 2007; 25(3): 203-08.

9. Gupta V, Datta P, Agnihotri N, Chander J. Comparative in vitro activities of seven new $\beta$-lactams, alone and in combination with $\beta$ lactamase inhibitors, against clinical isolates resistant to third generation cephalosporins. Braz J of infect Dis. 2006; 10(1): 22-25.

10.Prabhash K, Medhekar A, Biswas S, Kurkure $\mathrm{P}$, Nair R, Kelkar R. Comparison of in vitro activities of ceftazidime, piperacillintazobactam, and cefoperazone-sulbactam, and the implication on empirical therapy in patients with cancer. Ind J Cancer. 2009; 46:318-22.

11. Mohanty S, Singhal R, Sood S, Dhawan B, Das BK, Kapil A. Comparative in vitro activity of beta lactam/beta lactamse inhibitor combinations against gram negative bacteria. Ind J Med Res. 2005; 122:425-28.

12.Chitnis SV, Chitnis V, Sharma N, Chitnis DS. Current status of drug resistance among Gram-negative bacilli isolated from admitted cases in a tertiary care centre. J Assoc Phy Ind. 2003; 51:28-32.

13. Akova M. Sulbactam-containing betalactamase inhibitor combinations. Clin Microbiol Infect 2008; 14: 185-8.

14.Levin A.S. Multi-resistant Acinetobacter infections: a role for sulbactam combinations in overcoming an emerging worldwide problem. Clin Microbiol Infect 2002; 8(3): 144-53.

\section{How to cite this article:}

Subbalakshmi, E., H. Kalavathy Victor and Loganathan, K. 2020. Comparison of in vitro Activities of Ceftazidime-Tazobactam, Piperacillin-Tazobactam, and Cefoperazone-Sulbactam, and the Implication on Therapy in a Tertiary Care Teaching Hospital. Int.J.Curr.Microbiol.App.Sci. 9(01): 853-857. doi: https://doi.org/10.20546/ijcmas.2020.901.094 